\title{
THE CONCEPT OF THE PICTOGRAPHY AS A BACKGROUND FOR «FREE HANDS» WRITTEN BY PAUL ELUARD AND MAN RAY
}

\author{
Ivlieva Yuliia \\ Graduatestudent \\ ORCID 0000-0001-6997-7045 \\ Oles Honchar National University \\ 72 Gagarin St, 49000, Dnipro, Ukraine \\ ivleva.love1995@gmail.com
}

This article is part of a deeper study of the phenomenon of "picto-poetry" on the example of Paul Eluard and Man Ray's collection "Free Hands". The article proves the intermediality hypothesis of a collection based on the history of its creation, where the two types of arts not only complement each other, but merge into one, creating new forms and genres of French poetry. The purpose of the study is to determine the nature and imagery of the interaction of visual and verbal codes of artistic reality in the collection "Free Hands", as well as to identify the basic features of the composition "in four hands" on the example of the frontispiece of the collection. Research methods: descriptive, structural and semantic analysis methods that allow the identification of relationships between different sign systems (visual, plastic, etc.). The main feature of Paul Eluard and Man Ray's picto-poetic collection "Free Hands", in the preface to which P. Eluard outlined the principles of a single pictopoetic reproduction of the world, is the special principle of organizing the artistic space, when graphic realities also become "literary text", and the poetic text and its graphic "second voice" cannot be interpreted separately. The multi-layered and heterogeneous internal connections in the texts and graphics of authors serve as a form of reproduction of the underlying processes that take place in the human psyche, and the texts of Eluard create visual metaphors that resemble dreams. visual.

Keywords: "Picto-poetry", "in four hands", surrealism, synthesis, intermediality,

\section{La conception de pictographie comme un préalable à la création du recueil «Les Mains libres» de Paul Eluard et Man Ray}

Ivlieva Yuliia

étudiant diplômé

ORCID 0000-0001-6997-7045

Dnipro Université nationale Oles

72, Avenue Gagarin, 49000, Gonchar, Dnipro, Ukraine

ivleva.love1995@gmail.com

Cet article fait partie d'une étude plus approfondie du phénomène de la «pictopoésie» à la base de l'exemple du recueil de Paul Eluard et Man Ray "Les Mains libres ». L'article prouve l'hypothèse de l'intermédialité de ce recueil en se servant de l'histoire de sa création, où les deux types d'art ne se complètent pas seulement, mais fusionnent en un seul, en créant en même temps de nouvelles formes et de nouveaux genres de la poésie française. L'étude a pour objectif de déterminer la nature et les formes figuratives de l'interaction des codes visuels et verbaux de création de la réalité artistique dans le recueil "Les Mains libres", ainsi que d'identifier les caractéristiques de base de la composition "à quatre mains" sur l'exemple de frontispice du recueil. Les méthodes de la recherche: la méthode descriptive, l'analyse structurelle et sémantique qui permet de détecter la connexion entre les différents

(C) Ivlieva Yu., 2020 
systèmes sémiotiques (visuels, plastiques etc.) La propriété principale du recueil pictopoétique de Paul Eluard et Man Ray "Les Mains libres", dont le préambule reflète la vision de Paul Eluard sur des principes de la reproduction du monde picto-poétique intérgral, consiste en principe particulier d'organisation de l'espace artistique, lorsque des réalités graphiques deviennent elles aussi le "texte littéraire", et le texte poétique et sa "deuxième voix» ne peuvent pas être interprétés séparément. La composition multicouche et hétérogène des liens intérieurs dans le texte et la graphique des auteurs sert également de forme de la reproduction des processus en profondeur qui ont lieu dans la mentalité humaine, et les texte de Paul Eluard créent des métaphores visuelles qui ressemblent aux rêves et aux visions.

Mots-clés: "Picto-poésie", "à quatre mains", surréalisme, synthèse, intermédialité, visuel.

\section{Préface}

L'actualité du sujet de la recherche est déterminée par la nécessité de comprendre la contribution des surréalistes, en particulier de Paul Eluard et Man Ray, dans la création d'un langage artistique particulier, qui est une synthèse de différents arts dans le cadre d'un seul oeuvre qui élargissent les limites de la notion «texte artistique ». L'objectif de ce travail est d'identifier les traits de la poésie picto-poétique à la base de frontispice du recueil de Paul Eluard et Man Ray « Les Mains libres ».

Le but de l'étude détermine ses tâches spécifiques, telles que:

- présenter l'histoire de la création du recueil;

- cerner les traits de la composition "à quatre mains";

- déterminer la nature et les formes figuratives d'interaction entre les codes visuels et verbaux de la création de réalité artistique du recueil picto-poétique «Les mains libres».

Méthodes de recherche: les méthodes d'analyse descriptive, structurelle et sémantique qui identifient les relations entre les différents systèmes sémiotiques (visuels, plastiques, etc.).

Énoncé du problème. Le mouvement surréaliste français né au début du XXe siècle prône l'absence définitive de rationalité, la liberté de pensée et l'expression sous de nombreuses formes expérimentales, notamment celles picto-poétiques.

Les peintres surréalistes ont toujours rêvé de quelque chose de plus. Ils savaient que rien n'était suffisamment décrit dans l'art, rien n'était généré littéralement, ils cherchaient à libérer leur vision afin de joindre l'imagination à la nature, afin d'y aborder tout ce qui est possible comme réel. Ils savaient qu'il existait une autre réalité qui se manifestait dans la connexion insaisissable entre le spectateur et le visible. Cette envie de rendre visible d'autres réalités se manifeste clairement dans la collaboration des poètes et des peintres, et le recueil picto-poétique «Les Mains libres » en est la preuve, car l'idée de la composition du recueil consistait en combinant des deux types d'art: la poésie et la peinture, qui en semblant exister parallèlement, se connectent et ne peuvent pas être séparés.

On le voit clairement dans le contenu du recueil, qui se compose du frontispice, d'une préface, des poèmes et des dessins qui sont inséparables.

En s'appuyant sur l'histoire de la création du recueil, nous pouvons tracer le chemin que les peintres ont passé vers la création du phénomène de «picto-poésie», le chemin vers la synthèse des deux arts.

\section{Histoire de la création du recueil}

À l'époque le peintre talentueux Man Ray d'origine américaine était l'un des fondateurs de l'avant-garde visuelle française avant même son arrivée à Paris au début des années 1920. Protestaire par nature, Man Ray niait instinctivement toute influence artistique. Cependant, il a volontairement répliqué à l'appartition des techniques et des méthodes innovantes dans l'art, en les utilisant dans son oeuvre. La quintessence de la vision du monde du peintre se réduit au désir "de se libérer de toute restriction et de se concentrer sur la prise en conscience de l'instant, car c'est dans l'instant que la véritable nature de l'acte artistique se 
réalise" (Sers, 1982: 12-13). (Traduit pas moi -Y.I.). Les idées inhabituelles qui ont inspiré Man Ray pendant des réflexions pareilles sont réfractées à travers le prisme du rationalisme prononcé propre au peintre.

L'intérêt simultané pour l'art et la science, combiné à une attitude innovante envers le monde, l'a inspiré à expérimenter avec de différents matériaux et genres artistiques à la fois à part et en combinaison les uns avec les autres. Les œuvres de Man Ray sont quasiment toujours un jeu passionnant et continu entre les genres artistiques, les formes et les matériaux. Ainsi, sa photographie se rapproche de la peinture, la peinture cite des expériences photographiques, les œuvres d'un plan bidimensionnel sont parfois transformées optiquement en sculptures tridimensionnelles, et les "objets" connus sont inexplicablement transformés en peintures. En même temps le but de Man Ray n'est pas «l'imitation d'un langage artistique à l'aide d'un autre, mais d'effacement des frontières entre l'art majeur et l'art mineur et entre les différents médias» (Settford, 1944: 40) (traduit par moi- Y.I.).

C'est déjà à cette époque là que Paul Eluard tient une conférence dans laquelle il revient à l'idée que la poésie ne doit pas être une prérogative de plusieurs sciences privilégiées sélectionnées, elle doit être créée par tous et pour tous: «Si la poésie dont je parle s'exprime souvent par des mots, on ne saurait contester qu'aucun moyen d'expression lui soit refusé. Le surréalisme est un état d'esprit » (Dumas, Scheler, 1968: 515)

Et il cite comme exemple des peintres surréalistes qui ont la capacité de voir au-delà du monde réel et qui peuvent «joindre l'imagination à la nature " (Dumas, Scheler, 1968: 515) pour montrer qu' « il n'y a pas de dualisme entre l'imagination et la réalité, que tout ce que l'homme peut concevoir et créer provient de la même veine, est de la même matière que sa chaise, que son sang et que le monde qui l'entoure "(Dumas, Scheler, 1968: 516) Dans ces lignes, Paul Euard revient à l'idéalisme poétique de la pensée surréaliste.

Dans les années 1920, le poète est arrivé à la compréhension que «fondé sur le postulat qu'il n'y a pas de distinction entre la chose choisie et son image (mentale ou verbale), et que les images produites par la conscience ont autant de réalité que celles qui sont perçues» (Puff, 2013)

Selon les suppositions de Jean-Charles Gatau, c'est après cette conférence de l'été de 1936 que l'idée du recueil «Les Mains libres » a été inventée par les amis Mann Ray et Paul Eluard.

En 1936, Man Ray montre à Paul Eluard une série de dessins où les images du repos de la belle saison prévalaient («Burlesque», «La glace cassée») et des portraits pris au début de 1936.

Le 1er avril 1937, inspiré par ces images Paul Eluard publie douze poèmes dans la NRF. Man Ray donnera ensuite à Paul Eluard une quinzaine de dessins à compléter le recueil« Les Mains libres »: «L'attente », "L'arbre-rose », « La plage » et « Des nuages dans les mains », datant du séjour de Paul Eluard et Man Ray à Kernow en juillet 1937 chez Roland Penrose. En août, des amis ont rencontré Picasso et Dora Maar à Mougins.

Le recueil"Les Mains libres" est littéralement imprégnée de l'ambiance d'amitié et de créativité qui régnait en l'été des années 1936 et 1937, et se présente parfois comme un journal annuel avec la composition «à quatre mains ». Le recueil est en effet similaire au voyage méditerranéen, avec des noms de lieux exacts: «Avignon », « Les Tours d'Eliane » ou « La plage ».

\section{Aperçu du contenu principal}

Certains dessins sont similaires à des images d'avenues touristiques, comme «Le tournant », où l'on peut voir le paysage depuis les falaises devant lesquelles les amis passer à vitesse en voiture. 


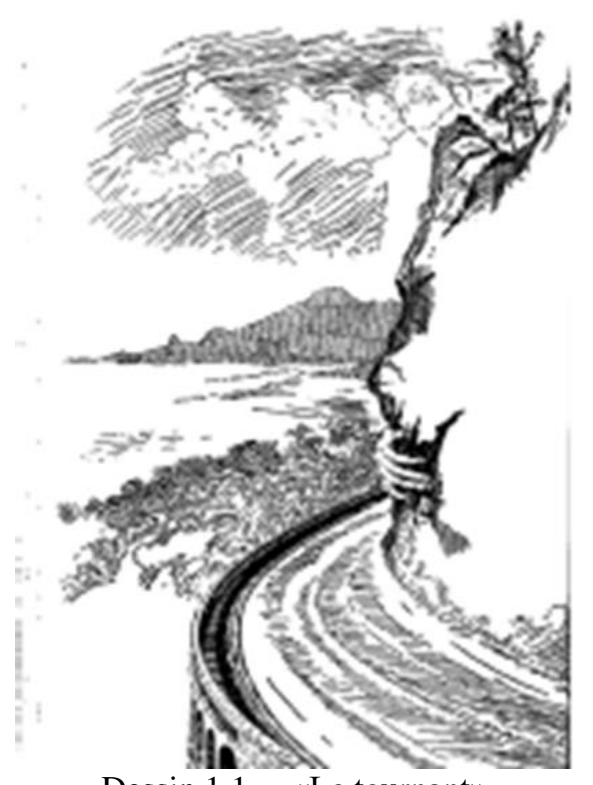

Dessin 1.1. - «Le tournant»

Enfin, un lien entre le recueil et le genre du journalistique est expliqué par Paul Eluar dans le double poème "Avignon », qui en un style de reportage capture l'ambiance de voyage:

\section{«Nous ne sommes restés qu'un moment à Avignon. \\ Nous avions hâte d'arriver à l'Isle-sur-Sorgue}

Où René Char nous attendait» (Ray, Eluard, 1937).

Cependant, une grande attention est consacrée ne pas seulement aux descriptions de stations de cure célèbres, mais aussi aux sites touristiques vus pendant les voyages, l'élément dominant du recueil est centré sur "les personnages » "des Mains libres », ce qui rend hommage à ceux qui ont été présents au sud de la France pendant l'été des années 1936-1937.

Ainsi, l'architectonique générale du recueil suggère de l'évaluer comme une sorte de l'hymne d'amitié et de créativité, à la fois graphique et poétique.

Le frontispice (un dessin ou un portrait au début d'un livre, à côté de la première page ou en haut de la page avant le texte (Dictionnaire de la langue ukrainienne, 1979) de recueil «Les Mains libres » contient une image d'un fragment des célèbres arches célèbres du Pont Saint- Bénézet du XIIIe siècle situé sur le Rhône à Avignon.

Avec l'image du pont, les auteurs ouvrent un élément symbolique, un chiffre, un message qui permet au spectateur ou au lecteur de passer, comme le disait Paul Eluard, "suppression des distances": du dessin au vers, d'un objet imaginaire à un autre - mais peutêtre d'abord de l'un ami à un autre, car ce frontispice reflète l'amitié de Man Ray et Paul Eluard.

Ainsi, à notre avis, ce dessin ouvre le recueil "Les Mains libres" sous le signe de l'amitié, de la coopération et de la créativité.

Sur cette image, le peintre Man Ray a marqué le pont Saint- Bénezet comme une femme nue géante allongée dessus, ressemblant à une déesse endormie qui, ayant les cheveux tombants, constitue par son corps une arche supplémentaire. La main droite de la femme repose sur le pont, sa main gauche est abaissée sur les deux chapelles situées derrière le pont: Saint-Bénezet et Saint-Nicolas. Ils occupent une tier gauche de l'image. Ses longs cheveux sont enfoncés dans l'eau de Rhône et son visage se reflète dans l'eau.

À notre avis, le réalisme figuratif combine les traits de conte de fées et de grotesque dans lequel l'érotisme donne la tonalité de base. Man Ray, que voulait-il souligner par ce dessin? De nombreux biographes et commentateurs s'accordent à dire qu'avec l'image du pont, l'auteur souhaitait exprimer l'idée d'une rencontre de deux arts, divisés entre deux mondes imaginaires, entre deux moyens d'expression. 
La confrontation du dessin et du texte enfonce le lecteur dans un espace mystérieux. Le spectateur peut d'abord comprendre les deux éléments à part en faisant appel à l'origine principale comme par réflexe. Il s'oriente avant tout à chercher les liens entre le texte et l'image.

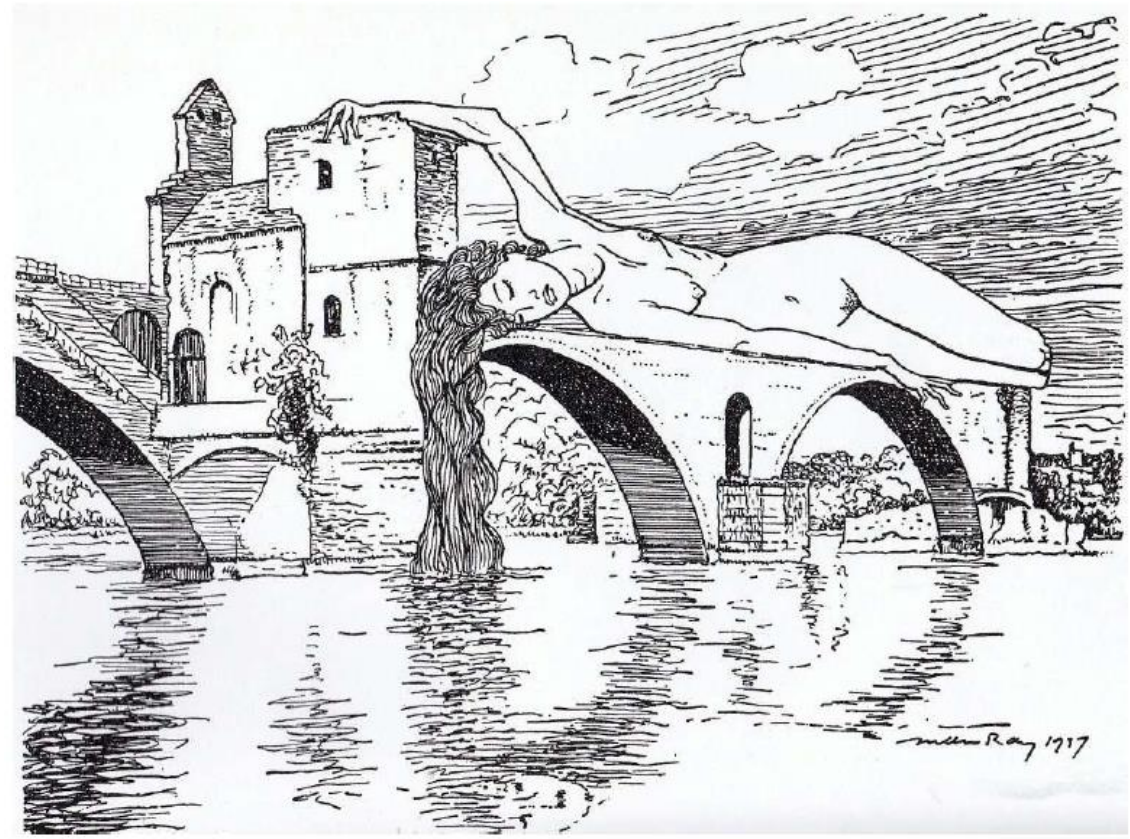

Dessin 1.2. - Man Ray - Le pont brisé, 1937

A l'image s'ajoute un commentaire verbal appartenant à la plume de de Paul Eluard: Le papier, nuit blanche. Et les plages désertes des yeux du rêveur. Le coeur tremble.

Le dessin de Man Ray: toujours le désir, non le besoin. Pas un duvet, pas un nuage, mais des ailes, des dents, des griffes.

Il y a autant de merveilles dans le fond d'un verre de vin que dans le fond de la mer. Il y a plus de merveilles dans une main tendue, avide que dans tout ce qui nous sépare de ce que nous aimons. Ne laissons pas perfectionner, embellir ce qu'on nous oppose.

Une bouche autour de laquelle la terre tourne. Man Ray dessine pour être aimé (Ray, Eluard, 1937).

Dans la préface «des Mains libres», l'association surréaliste des mots devient immédiatement apparente. Il contient des mots qui apparaissent dans le contenu du recueil. Le «papier» fait référence à l'écriture et au dessin. "La nuit blanche" à l'amour. "Les plages désertes » font appel à la plage principale. "Les yeux du rêveur" contiennent une image métaphorique des yeux et de la magie.

La bouche (les lèvres) est l'un des symboles surréalistes préférés. Malgré le caractère allégorique évincé inhérent à ce genre, la bouche féminine a provoqué chez les peintres surréalistes une série de simples d'associations: sensualité, mystère, séduction.

La notion de «miracle» fait référence à une confrontation surréaliste entre la réalité objective et l'univers intérieur. «Les miracles » se réfèrent à ce que le lecteur verra, trouvera, interprétera. Ce mot prend une couleur particulière dans le contexte du surréalisme (la recherche du merveilleux, du fabuleux qui dépasse la réalité, le désir l'accomplissement de soi). «Désireux» peut aussi signifier un désir de retrouver le sens que le lecteur peut ressentir. «Désir » rappelle le sujet central de ces œuvres érotiques. «Les mains » signifient le motif reproductible annoncé dans le titre du recueil.

Les dessins « des Mains libres » recèlent une énergie sexuelle et indiquent que l'artiste y attache une grande importance: «le dessin de Man Ray: toujours le désir, non le besoin». Le besoin est physiologique (alimentation, sexualité ...), l'envie de création artistique. La libido lance le travail de l'inconscient, et la créativité l'inscrit dans le symbolisme social et culturel, 
dans son imagination. Les rêves, les fantasmes, l'acte créatif, œuvre d'art en soi-même sont les produits du désir. Et le poète crée, rêve, fantasme sur le désir de l'artiste plus que sur l'objet de son propre désir. C'est ainsi que le poète perçoit le monde. Il s'associe au peintre dans l'équivalence, dans une interchangeabilité qui lui permet de vivre, d'aimer et de créer par le remplacement ou du moins par sa médiation.

En effet, Paul Eluard, comme aussi Man Ray, se concentre sur ce qui les unit soit le désir mentionné dans deuxième paragraphe de la préface au sens figuré dans le frontispice de Man Ray sous l'image d'une femme nue géante allongée sur un pont. L'image de la Femme a une grande importance pour la poésie du surréalisme, étant l'incarnation des racines naturelles, les forces motrices de l'être, le symbole de deux sources universelles - une grande source destructrice et celle créatrice.

Étant le partenaire spirituel et physique de l'homme, la Femme est l'initiatrice de sa lutte (d'un homme) pour la libération intérieure et la médiatrice entre sa conscience rationnelle (de l'homme) et l'essence de l'être. En le même temps, l'amour selon la vision du monde surréaliste est la seule force humaine capable de franchir les portes de fer de la langue et du bon sens.

Par conséquent, un homme n'est capable de création artistique que dans une seule impulsion amoureuse et dans une alliance harmonieuse avec la Femme. À cet égard, l'image globale de la femme inspire non seulement les surréalistes à créer des œuvres, mais remplit souvent presque tout espace du texte artistique.

Ainsi, la Femme, l'Amour et l'Erotisme sont vraiment le principal pont fondamental entre les deux artistes. Ces lignes peuvent en fait servir d'épigraphe au recueil, car elles résument son opinion dominante. Le surréalisme affirme la relativité du monde et de ses valeurs. Il n'y a pas de frontières entre le bonheur et le malheur, la personnalité et la société. Le chaos du monde provoque également le chaos de la pensée artistique, ce sont les principes de la poésie du recueil «Les Mains libres » et de l'esthétique du surréalisme.

\section{Conclusions et suggestions.}

On arrive à la conclusion que, essentiellement, toutes les esquisses picto-poétiques du recueil «Les Mains libres » sont basées sur une combinaison des concepts insociables, sur la destruction des relations spatiales objectives. L'inclusion des objets visuels dans le texte poétique permet de monter au niveau de la contemplation, de l'observation, étant la source de toute sensibilisation. L'utilisation de la composante visuelle, représentée sous forme de dessin, est principalement associée à la tâche du poète, qui se résume à essayer de s'exprimer, d'exprimer son idée dans l'espace linguistique minimal, sans perdre le contenu informatif du texte.

Il est à noter que la composante visuelle introduite dans le texte poétique y occupe une place dominante. Dans le texte poétique classique, un seul canal de communication, précisément par le texte écrit. Dans le vers pictographique, avec l'introduction de la composante visuelle, un deuxième canal de communication s'y rajoute, un canal visuel. Pour cette raison, la partie verbale du texte est réduite, car l'image mentale formée dans l'esprit du poète peut être exprimée à l'aide de la composante visuelle, qui est le dessin.

L'objectif de la collaboration créative est de changer la relation traditionnelle entre le texte et l'image. De plus, les illustrations de Man Ray et les poèmes de Paul Eluard offrent l'occasion d'opposer les deux façons de présenter et de créer une image du monde et de trouver de nouvelles interprétations pour le lecteur et le spectateur.

Donc, à notre avis, la tâche artistique des auteurs est de découvrir de nouvelles significations, d'explorer des mondes cachés ainsi que de laisser au lecteur la possibilité de faire ses propres interprétations et de telle manière participer à la création du recueil.

Réferences

P. Sers, 1982 - P. Sers (1982). Man Ray and the Avant-garde. London: Thames and Hudson. 
D. Settford, 1944 - D. Settford (1944). Man Ray's Man Rays. New-York: West Palm Beach.

1. Marcelle Dumas et Lucien Scheler (1968). Paul Eluard. Oeuvres completes. Paris: Gallimard.

2. Jean-François Puff (2013). Du livre ouvert au livre fermé: Éluard à rebours. Fabula. La recherché en literature. Available at:

http://www.fabula.org/colloques/document2244.php

3. Man Ray, Paul Eluard (1937). Les Mains libres. Le contexte de création. Available at: https://www.lettresvolees.fr/eluard/autoportrait.htm

4. Slovnik ukrayins `koyi movi: v 11 tomakh (1979). [Ukrainian dictionary] Kiyv: «Naukova dumka».

\section{REFERENCES}

1. P. Sers (1982). Man Ray and the Avant-garde. London: Thames and Hudson.

2. D. Settford (1944). Man Ray's Man Rays. New-York: West Palm Beach.

3. Marcelle Dumas et Lucien Scheler (1968). Paul Eluard. Complete Works. Paris: Gallimard. [in French].

4. Jean-François Puff (2013). From open book to closed book: Eluard conversely. Fabula. Literature research. Available at: http://www.fabula.org/colloques/document2244.php [in French].

5. Man Ray, Paul Eluard (1937). Free Hands. The context of creation. Available at: https://www.lettresvolees.fr/eluard/autoportrait.htm [in French].

6. Dictionary of the Ukrainian language: in 11 volumes. (1979). Kiyv: «Naukova dumka». [in Ukrainian].

Received: 25 May, 2020 
